0.001) and coagulopathy/ thrombocytopenia, PRISM III $\geq 10$, renal and hepatic failure, hypotension, and heart failure/ arrhythmia was found to be associated with the development of GIB in critically ill children $(\mathrm{p}<0.05)$.

Conclusions GIB is a serious concern for PICU clinicians and intensivists are confused about the conflicting evidence supporting prophilaxis. We believe that prophylaxis could be beneficial for mechanically ventilated children. Also trauma, coagulopathy/ thrombocytopenia, PRISM III $\geq 10$, renal and hepatic failure, hypotension, and heart failure/arrhythmia must be kept in mind as risk factors requiring attention in PICU setting.

\section{PO-0139 WILSON'S DISEASE: TEN YEARS RETROSPECTIVE EXPERIENCE AT NATIONAL LIVER INSTITUTE, EGYPT}

E Salama, E Behairy, A Ahmed, M Nermin, M Ahmed. Pediatric Department, Menoufia University, Cairo, Egypt

\subsection{6/archdischild-2014-307384.802}

Background and aims Wilson's disease (WD) is a rare, inherited, genetic disorder of copper metabolism. Our aims to determine common clinical presentations, laboratory findings, diagnostic methods and long term outcome in Egyptian patients.

Methods All medical records, between 2000 and 2010 in the paediatric hepatology department, were reviewed. Detailed follow-up data of the disease had been collected for each patient. Serum ceruloplasmin, liver function tests and other routine laboratory investigations. Slit lamp examination for Kayser Fleisher rings and 24-hour urine for copper before and after penicillamine challenge were done. Percutaneous liver biopsy also was performed in most patients.

Results The most significant hepatic presentation was jaundice and Kayser Flisher rings. The most significant laboratory findings were, copper excretion after challenge with depencillamine $(1546.57 \pm 99.55 \mu \mathrm{g} / \mathrm{Dl})$ and decrease of mean ceruloplasmin concentration $(13.8 \pm 2.38 \mathrm{mg} / \mathrm{dl})$ below $20 \mathrm{ug} / \mathrm{dl}$. There were significant increase of albumin and significant improvement of prothrombin time after treatment.

Conclusion Kayser Flisher rings, urinary copper excretion and low serum ceruloplasmin were considered sufficient to establish the diagnosis of WD. Liver biopsy may be needed for confirmation of the diagnosis and to assess the extent and severity of the disease.

\section{PO-0140 WHETHER ANTIMICROBIAL THERAPY AFFECT FECAL EXCRETION TIME IN PAEDIATRIC PATIENTS OF NONTYPHOID SALMONELLOSIS WITH DIFFERENT SEVERITY}

YT Shen, IF Huang, HH Hu, MF Chang, SK Sheu. Department of Pediatrics, Kaohsiung Veterans General Hospital, Kaohsiung, Taiwan

10.1136/archdischild-2014-307384.803

Objective To investigate whether the administration of antibiotics affect faecal excretion time in paediatric salmonellosis with different severity.

Methods Children with nontyphoid salmonellosis admitted to Kaohsiung Veterans General Hospital, Taiwan who consented to receive consecutive stool cultures every 4-7 days till two consecutive negative results between 2005 and 2013 were enrolled. Faecal excretion time was defined as the timeframe of the first positive stool culture and the first of two consecutive negative results. The Severity Score was used to stratify the patients as the severe, moderate and mild group according to their fever days before admission, band cells in peripheral blood and Creactive protein. Patients were classified into no antibiotics (mild disease without antibiotic), concordant (severe patients receiving antibiotics active in vitro) and inappropriate therapy group (mild patients receiving antibiotics or severe patients receiving antibiotics resistant in vitro).

Results 53 patients were enrolled. Compared with no antibiotic group ( $\mathrm{n}=28)$, the concordant group $(\mathrm{n}=11)$ had comparable faecal excretion time (12.36 vs 9.79 days), fever days after admission and hospital stay $(2.45$ vs 2.25 ; 6.45 vs 6.39 days). The inappropriate group $(\mathrm{n}=14)$ had significantly longer faecal excretion time ( 24.50 vs 9.79 days), hospital stay ( 8.50 vs 6.39 days) and higher platelet count (333.43 vs $269.82 \times 1,000 /$ $\mathrm{mm} 3$ ) than no antibiotics group. With further multiple regression analysis, only inappropriate antibiotic use affected significantly $(\mathrm{p}=0.008)$ on faecal excretion time.

Conclusions Antibiotics used in severe children with salmonellosis didn't prolong faecal excretion time, but inadequate use of antibiotics prolonged faecal excretion time in Salmonellosis.

\section{PO-0141 OVERVIEW ON THE PHYSIOLOGY OF LONGITUDINAL INTESTINAL LENGTHENING AND TAILORING IN SHORT BOWEL SYNDROME}

${ }^{1} \mathrm{~S}$ Siminas, ${ }^{2} \mathrm{R}$ Coletta, ${ }^{2} \mathrm{M}$ Baath, ${ }^{2} \mathrm{~A}$ Morabito. ${ }^{1}$ Paediatric Surgery Department, Manchester Children's Hospital, Manchester, UK; ${ }^{2}$ Paediatric Autologous Bowel Reconstructive Unit, Manchester Children's Hospital, Manchester, UK

\subsection{6/archdischild-2014-307384.804}

Background Multidisciplinary treatment of short bowel syndrome (SBS) has been a success story for paediatric surgery. Longitudinal intestinal lengthening and tailoring (LILT) is the archetypal autologous gastrointestinal reconstruction procedure, but despite it being performed routinely in intestinal rehabilitation departments, the physiological basis behind it is poorly understood. We attempt to analyse the features that improve intestinal adaptation and offer a LILT theoretical model.

Methods Based on our clinical experience on 59 LILT procedures over 30 years, we set up a concise theoretical model that describes post-LILT bowel adaptation in a holistic way.

Results Intestinal adaptation is affected by many factors, including remaining bowel length, the presence of the ileocaecal valve and colon, underlying disease process, nutritional status, hepatobiliary function, and bacterial flora. Lengthening techniques do not affect the absorptive surface, although changes in the microvilli have been observed in animal models. Dilated dysmotile intestinal segments benefit from tapering because reduction in the intestinal lumen calibre allows better peristalsis, as demonstrated by improvements in intestinal transit time. Tapering also helps prevention of stasis and subsequently reduction of bacterial overgrowth. Additionally, reduction in bowel diameter decreases the volume to surface ratio, theoretically allowing for more effective contact of the chime with the absorptive surface.

Conclusions LILT has become an essential component in the management of SBS. The physiological principles described above provide a theoretical basis that explains the absorptive advantage offered by the LILT. Further research is necessary to quantify the effect of these procedures on the microscopic and hormonal levels. 